Original

\title{
Experiencia en reconstrucción postraumática de uretra posterior en niños. Hospital Universitario San Vicente de Paúl. Medellin 1987-2007
}

\author{
Jorge A. Martínez Montoya, Natalia M. Tascón Acevedo \\ Sección de Cirugía y Urología Pediátrica. Facultad de Medicina. Universidad de Antioquia. Medellín. Colombia.
}

\begin{abstract}
Resumen
Objetivo: Explorar el desenlace de los diferentes abordajes quirúrgicos para el tratamiento del trauma de uretra en los pacientes pediátricos, evaluando en cada caso, sus secuelas tales como son la estenosis, la incontinencia y la impotencia.

Métodos: Se revisaron en forma retrospectiva las historias clínicas de 43 pacientes que consultaron al servicio de urgencias del Hospital Infantil San Vicente de Paúl, con ruptura de la uretra posterior, desde el año 1987 hasta el 2007. En estos pacientes se evaluaron los datos demográficos, el tipo de procedimiento quirúrgico realizado y las complicaciones tempranas y tardías.

Resultados: El tiempo promedio de seguimiento fue de 30.6 meses, y el promedio de edad, al momento del trauma, fue de 7,7 años. Todos los pacientes fueron del sexo masculino y todos con ruptura de uretra posterior. En 27 pacientes se realizó uretroplastia primaria (62,8\%), en 13 se realizó cistostomía y reparo tardío $(30,2 \%)$ y en 3 pacientes se realizaron otros procedimientos $(7 \%)$.

El 39,5\% de los pacientes presentaron en algún momento del seguimiento complicaciones. En el grupo de pacientes estudiados, las complicaciones fueron estenosis uretral en 26 pacientes $(60,5 \%)$, retención urinaria por obstrucción en 10 pacientes $(23,3 \%)$, la incontinencia en 10 pacientes $(23.3 \%)$ y la impotencia en 7 pacientes $(16,3 \%)$.

Los pacientes en los cuales se realizó uretroplastia primaria presentaron un riesgo significativamente menor de desarrollar infección, obstrucción y estenosis.

Aquellos pacientes con fractura de pelvis asociada al trauma uretral, tuvieron un riesgo significativamente mayor de desarrollar estenosis e impotencia $(\mathrm{p}<0,05)$.

Conclusión los diferentes abordajes quirúrgicos utilizados presentan tasas de complicación y de morbilidad a mediano plazo similares. El procedimiento de elección debe ser individualizado de acuerdo a las características del paciente, la extensión del daño uretral, las lesiones asociadas y la experiencia del cirujano en el manejo del trauma uretral. En nuestro estudio encontramos que los pacientes en los cuales se realizo uretroplastia primaria presentaron un menor riesgo de infección, estenosis y obstrucción.
\end{abstract}

Palabras clave: Trauma de uretra posterior. Niños. Uretroplastia primaria. Cistostomía. Reparo tardío.

\section{Surgical approach of traumatic urethral injury in children. Experience at San Vicente of Paul Universitary Hospital. Medellin 1987-2007}

\section{Abstract}

Objective: In order to evaluate the efficacy of different surgical techniques for the correction of traumatic lesions of the urethra, we performed a retrospective study in those patients, and evaluated different complications such as postsurgical stenosis of the urethra, incontinence and impotence (erectile dysfunction).

Methods: A retrospective study was conducted, reviewing the clinical charts of 43 patients admitted to the San Vicente of Paul Hospital, with diagnosis of traumatic rupture of the posterior urethra from 1987 to 2007 . We analyzed different demographic data, type of surgical correction, early and late complications.

Results: The average age of the patients was 7.7 years, the average follow up was 30.6 months, and all the patients were male with a posterior urethral rupture.

27 Patients underwent a primary urethral repair (63\%), 13 patients underwent a cistostomy with later urologic reconstruction (30\%), in 3 patients $(7 \%)$ other surgical procedures were made.

Overall complication rate was $39.5 \%$. These complications were: Urethral stenosis, 26 patients (60.5\%), urinary retention secondary to obstruction, 10 patients (23.3\%), incontinence 10 patients (23.3\%) and impotence 7 patients (16.3\%).

Patients treated with a primary urethral repair presented a significantly less development of infection, obstruction and stenosis. (p<0.05). Patients with pelvis fracture associated to urethral trauma had a significant higher risk of developing stenosis and impotence. ( $\mathrm{p}<0.05)$. Conclusions: Both different surgical techniques compared showed a similar complication and morbidity rates in middle follow up. Each procedure should be selected according to clinical condition of the patient, the extension of the urethral damage, the associated traumatic lesions and the surgeon`s expertise.

In our searched patients, treated with a primary urethral repair we found a significantly less development of infection, obstruction and stenosis.

Keywords: Trauma of urethra posterior. Children. Primary urethral repair. Cistostomy with later urologic reconstruction. 
T a ruptura de la uretra en niños constituye un Lreto para el cirujano pediátrico, su incidencia se estima en 1 de cada 2000 niños admitidos por lesiones traumáticas, y corresponden al 3,4\% de los niños que tienen un trauma genitourinario. El trauma de uretra se asocia a complicaciones graves para el paciente que incluyen la incontinencia urinaria, la disfunción eréctil y la formación de estenosis.

En las ultimas dos décadas el desarrollo de las diferentes técnicas de uretroplastia ha mejorado las perspectivas de los pacientes con lesiones de uretra, en la actualidad, con, tasas de éxito en la reparación de la uretra cercanas al 90\%. Sin embargo, el tratamiento de las lesiones postraumáticas de uretra continua siendo un tema controvertido, con relación al abordaje quirúrgico inicial, y el momento optimo para la reconstrucción quirúrgica, así como el manejo de las complicaciones que se derivan del mismo.

Hay pocas revisiones en la literatura mundial que evalúen las técnicas para la reconstrucción de uretra en pacientes pediátricos. Algunos autores están a favor del reparo uretral primario y consideran que hay disminución de la estenosis uretral en estos pacientes ${ }^{1,2}$. Mientras otros favorecen una cistostomía inicial con un reparo uretral tardío, encontrando en estos pacientes una disminución de la incidencia de incontinencia e impotencia ${ }^{1,2}$.

Por otra parte hay quienes recomiendan el paso retrogrado de una sonda uretral, a manera de tutor, sin anastomosis ${ }^{3,4}$

A pesar de los estudios publicados, hasta la fecha no existe claridad, acerca de cual puede ser el manejo mas indicado, por ofrecer los menores riesgos de estenosis, impotencia e incontinencia para los pacientes pediátricos.

El objetivo del estudio es evaluar la eficacia de los diferentes abordajes para el tratamiento del trauma de uretra en pacientes pediátricos, en términos de sus principales secuelas tales como son la estenosis, la incontinencia y la impotencia.

\section{MATERIALES Y MÉTODOS}

Se realizó un estudio de tipo retrospectivo, de corte transversal y descriptivo.

Se incluyeron todos los pacientes menores de 12 años que presentaron trauma de uretra y que fueron valorados por Cirugía Pediátrica del Hospital Universitario San Vicente de Paúl (HUSVP) de Medellín, entre el 1 de enero de 1987 y el 31 de diciembre de 2007.
Los registros se obtuvieron de la base de datos del servicio de cirugía pediátrica, se revisaron los registros de 116 pacientes sometidos a procedimientos uretrales en el periodo de tiempo mencionado, de ellos 44 pacientes presentaban lesiones uretrales de tipo traumático, 43 pacientes con trauma de uretra posterior y un paciente con trauma de uretra anterior, el cual se excluyo del estudio.

La selección del procedimiento quirúrgico inicial realizado, dependía de la decisión del cirujano a cargo del caso, de acuerdo a los hallazgos clínicos e imaginologicos encontrados en cada paciente.

La recolección de los datos se realizo en un formulario que incluyo: identificación del paciente, datos demográficos, mecanismo del trauma, localización del trauma uretral, presencia de otras lesiones asociadas, puntaje de trauma, técnica quirúrgica empleada, días de estancia hospitalaria, complicaciones tempranas, complicaciones tardías, necesidad de reintervenciones y de dilataciones de uretra posterior al procedimiento quirúrgico.

Para el proceso de tabulación y análisis se creo una base de datos con el programa SPS 11.0, con análisis unívariado y Bivariado. Para el procesamiento de textos y tablas, se utilizo el programa Microsoft office 2007. Las variables cuantitativas se analizaran sacando de cada una de ellas la media, las desviaciones estándar, el rango y la moda; cuando no hubo una distribución normal se utilizó mediana. Para las variables cualitativas se calcularon frecuencias y proporciones con la prueba de Chi cuadrado de Pearson.

Basados en la resolución 8430 de 1993 del ministerio de salud, esta investigación se clasifico según el articulo 11 como una investigación sin riesgo. Los datos fueron tomados de las historias clínicas de los pacientes por profesionales con conocimiento y experiencia para proteger al paciente.

\section{RESULTADOS}

Durante el periodo de estudio 1 de enero de 1987 a 31 de diciembre de 2007, se trataron en el HUSVP, 43 pacientes de sexo masculino con trauma de uretra posterior. La edad promedio fue de 7,7 años, 33 pacientes (76\%) se encontraban en edad escolar. El mecanismo de la lesión fue traumático en el 97,7\% de los pacientes y fue iatrogénico en el 2,3\% de los pacientes. Se trato de un paciente con lesión de uretra posterior secundaria a corrección de una malformación ano rectal alta (Tabla 1). 
Tabla 1. Datos demográficos. Experiencia en reconstrucción postraumática de uretra en niños. HUSVP Medellín 1987-2007

\begin{tabular}{llcc}
\hline Variable & & $\mathbf{N}^{\mathbf{0}}$ & $\mathbf{\%}$ \\
\hline Grupos de edad & Lactantes & 6 & 14,0 \\
& Preescolares & 4 & 9,3 \\
& Escolares & 33 & 76,7 \\
& Total & $\mathbf{4 3}$ & $\mathbf{1 0 0 , 0}$ \\
& & 42 & 97.7 \\
Mecanismo de Trauma & Traumático & 1 & 2.3 \\
& Iatrogénico & $\mathbf{4 3}$ & $\mathbf{1 0 0 , 0}$ \\
& Total & & \\
Índice de severidad & Leve (puntaje mayor de 8) & 25 & 58,1 \\
& Moderado (puntaje de 0 a 8) & 14 & 32,6 \\
& Sin Dato & 4 & 9,3 \\
& Total & $\mathbf{4 3}$ & $\mathbf{1 0 0 , 0}$ \\
\hline
\end{tabular}

El 93\% (40 pacientes) presentaban lesiones asociadas al trauma de uretra y de ellos el 76.7\% (33 pacientes) presentaron fractura de pelvis. En el 58\% de los pacientes con lesiones traumáticas el índice pediátrico de trauma fue leve (puntaje mayor de 8) (Tablas 1 y 2).

En 27 casos (62\%), el procedimiento quirúrgico inicial fue la uretroplastia primaria, en 5 de estos pacientes fue necesaria adicionalmente la reparación del cuello vesical. En 13 casos (30\%) el manejo quirúrgico inicial fue con cistotomía y reparo uretral tardío. En 3 pacientes (7\%) se realizaron otros procedimientos: En un paciente cistostomía y cateterismo retrogrado, en otro paciente, con lesión parcial de uretral, paso de sonda vesical únicamente y en un paciente de 3 meses de edad con lesión iatrogénica de la uretra, que se manifestó como fístula perineal, se realizo inicialmente una colostomía de doble boca y posteriormente uretroplastia tardía.

Tabla 2. Distribución porcentual de lesiones traumáticas asociadas

\begin{tabular}{lcc}
\hline Lesiones Asociadas & $\mathbf{N}^{\mathbf{0}}$ & $\mathbf{\%}$ \\
\hline Fractura de pelvis & 33 & 76,7 \\
Trauma de genitales & 5 & 11,6 \\
Lesión de víscera sólida abdominal & 3 & 7,0 \\
Lesión de víscera hueca abdominal & 15 & 34,9 \\
Fractura en extremidades inferiores & 14 & 32,6 \\
Trauma de tórax & 1 & 2,3 \\
Lesión de tejidos blandos & 2 & 4,7 \\
Fractura en extremidades superiores & 3 & 7,0 \\
Trauma Craneoencefálico & 2 & 4,7 \\
\hline
\end{tabular}

La severidad del trauma no influyo en el tipo de procedimiento a realizar en la conducta inicial.

Siete pacientes presentaron complicaciones tempranas. Las cuales se muestran en la Tabla 3.

En 2 pacientes no se encontró información sobre complicaciones tempranas.

Durante la primera hospitalización se reintervinieron 5 pacientes (11,6\%), 3 pacientes por desplazamiento de la cistostomía, y 2 pacientes que requirieron laparotomía exploradora uno por obstrucción por bridas realizándose liberación de adherencias y resección intestinal con anastomosis termino- terminal y otro paciente por trauma renal bilateral asociado, que necesito polectomía renal superior izquierda y drenaje de urinoma.

Los días de estancia hospitalaria fueron en promedio de 35.9, SD 3.4 (3-158).

En 35 pacientes $(81 \%)$, se realizó calibración uretral y el número promedio de dilataciones por paciente con estenosis uretral fue de 4.5, DE 3.2 (1-12).

El tiempo de seguimiento promedio fue de 30,5 meses (DE 44,1), durante este periodo de tiempo se evaluó la presencia de estenosis, infección, sangrado, obstrucción, incontinencia e impotencia como complicaciones tardías. La complicación mas frecuentemente encontrada fue la estenosis uretral en el $60,5 \%$ de los pacientes, seguida en frecuencia por la incontinencia y la obstrucción en un $23,3 \%$ cada una (Tabla 3).

Tabla 3. Complicaciones tempranas y tardías.

\begin{tabular}{llcc}
\hline Variable & & $\mathbf{N}^{\mathbf{0}}$ & $\%$ \\
\hline $\begin{array}{l}\text { Complicaciones } \\
\text { tempranas }\end{array}$ & $\begin{array}{l}\text { Complicaciones } \\
\text { relacionadas con } \\
\text { las lesiones asociadas }\end{array}$ & 3 & 7,0 \\
& $\begin{array}{l}\text { Estenosis de uretra } \\
\text { Urinoma e hidronefrosis }\end{array}$ & 2 & 4,7 \\
& bilateral & 1 & 2,3 \\
& Infección del tracto & & \\
urinario & 1 & 2,3 \\
Complicaciones & Estenosis & 26 & 60,6 \\
tardías & Obstrucción & 10 & 23.3 \\
& Incontinencia & 10 & 23,3 \\
& Impotencia & 7 & 16.3
\end{tabular}


En este trabajo se exploró la relación entre el tipo de procedimiento quirúrgico inicial y las complicaciones posteriores.

Los pacientes en los cuales se realizó uretroplastia primaria presentaron un riesgo significativamente menor de desarrollar infección, obstrucción y estenosis como se observa la Tabla 4.

Los pacientes con fractura de pelvis tuvieron un riesgo significativamente mayor de desarrollar estenosis e impotencia $(\mathrm{p}<0,05)$, pero no se comprobó en el caso de la incontinencia ninguna relación. $(\mathrm{p}=0,07)$.

\section{DISCUSIÓN}

Si bien el traumatismo uretral no amenaza la vida del paciente pediátrico, es sabido que, a largo plazo, puede traer consecuencias devastadoras y persistentes para el paciente, por ello su manejo quirúrgico sigue siendo un reto para el cirujano.

Al analizar los datos demográficos y comorbilidades de nuestra población de estudio se encontró similitud con los datos reportados por la literatura mundial, en general el mecanismo de lesión es traumático y los accidentes de transito ocupan la primera causa de lesión. En el grupo de pacientes revisados, el 97,7\% de las lesiones fueron traumáticas y solo el $2,35 \%$ iatrogénico ${ }^{1-3}$. La edad promedio fue de 7,7 años y la asociación de trauma uretral con fractura de pelvis se presento en el $76,7 \%$ de los pacientes. En la literatura medica, se ha reportado que fracturas de pelvis en los niños tienden a ser inestables y se asocian con un desplazamiento severo y permanente de la uretra posterior, con porcentajes de asociación del 95\% ${ }^{4}$. En nuestros pacientes, el porcentaje de asociación de trauma de uretra y fractura de pelvis solo fue de $76,6 \%$, pues 10 pacientes con trauma de uretra no presentaron fractura pelvis, sin embargo, estos pacientes presentaron lesiones severas de los tejidos blandos pélvicos, con avulsiones y luxación sacroliaca.

Encontramos una relación estadísticamente significativa $(\mathrm{p}<0,05)$ entre la presencia de fractura y la aparición de estenosis e impotencia como complicaciones tardías; En la literatura la incidencia de impotencia después de una lesión de uretra asociada a fractura pélvica varia entre el 2,5 al 62\% dependiendo de el grado de la misma los pacientes con lesión de uretra y fracturas pélvicas tipo IV tiene un porcentaje de complicación cercano al $28 \% \%^{2,5}$.

Nuestros pacientes presentaron un índice de trauma pediátrico leve a moderado sin mortalidad asociada en la literatura la mortalidad del trauma de uretra en niños se asocia con la presencia de fractura de pelvis y lesión abdominal y es cercano al $8 \%{ }^{2}$.

El abordaje quirúrgico sigue siendo motivo de controversia la realización de la uretroplastia primaria en las lesiones uretrales fue propuesto por primera vez en 1929 por Young inicialmente la téc-

Tabla 4

\begin{tabular}{|c|c|c|c|c|c|c|}
\hline Complicaciones & & $\begin{array}{l}\text { Uretroplastia } \\
\text { primaria }\end{array}$ & $\begin{array}{l}\text { Cistostomía y } \\
\text { reparo tardió }\end{array}$ & $\mathbf{p}$ & OR & IC $95 \%$ \\
\hline Infección & $\begin{array}{l}\mathrm{Si} \\
\text { No } \\
\text { Total }\end{array}$ & $\begin{array}{c}5 \\
20 \\
25\end{array}$ & $\begin{array}{c}8 \\
6 \\
14\end{array}$ & 0,01 & 0,188 & $(0,044-0,793)$ \\
\hline Sangrado & $\begin{array}{l}\mathrm{Si} \\
\text { No } \\
\text { Total }\end{array}$ & $\begin{array}{c}0 \\
25 \\
25\end{array}$ & $\begin{array}{c}1 \\
13 \\
14\end{array}$ & 0,176 & - & - \\
\hline Obstrucción & $\begin{array}{l}\mathrm{Si} \\
\text { No } \\
\text { Total }\end{array}$ & $\begin{array}{c}3 \\
22 \\
25\end{array}$ & $\begin{array}{c}7 \\
7 \\
14\end{array}$ & 0,009 & 0,136 & $(0,028-0,674)$ \\
\hline Estenosis & $\begin{array}{l}\mathrm{Si} \\
\text { No } \\
\text { Total }\end{array}$ & $\begin{array}{l}14 \\
11 \\
25\end{array}$ & $\begin{array}{c}12 \\
2 \\
14\end{array}$ & 0,05 & 0,212 & $(0,39-1,152)$ \\
\hline Incontinencia & $\begin{array}{l}\mathrm{Si} \\
\text { No } \\
\text { Total }\end{array}$ & $\begin{array}{c}6 \\
19 \\
25\end{array}$ & $\begin{array}{c}4 \\
10 \\
14\end{array}$ & 0,7 & 0,789 & $(0,180-3,464)$ \\
\hline Impotencia & $\begin{array}{l}\mathrm{Si} \\
\text { No } \\
\text { Total }\end{array}$ & $\begin{array}{c}6 \\
19 \\
25\end{array}$ & $\begin{array}{c}1 \\
13 \\
14\end{array}$ & 0,18 & 4,105 & $(0,441-38,234)$ \\
\hline
\end{tabular}


nica se describió por vía perineal y posteriormente varios estudios han recomendado el abordaje transpúbico, el cual es el mas recomendado por algunos autores en los pacientes pediátricos ${ }^{6}$.

Entre las ventajas de la uretroplastia primaria están obtener un adecuado lumen uretral, la colocación de tutores por corto tiempo y minimizar las complicaciones de la cicatrizaron prolongada ${ }^{6,7}$

Por otro lado, el reparo tardío de las lesiones uretrales por otro lado permite controlar las condiciones asociadas del paciente antes de la realización del procedimiento quirúrgico y aunque se considera el tratamiento de elección en el paciente adulto en el paciente pediátrico se ha asociado con estenosis principalmente si la uretra no esta bien posicionada o existen lesiones de gran longitud ${ }^{4,7}$. La presencia de una cistostomía a largo plazo se asocia con infecciones de tracto urinario, litiasis vesical e incomodidad por la sonda ${ }^{7}$.

En nuestro estudio el $62,5 \%$ de los pacientes se sometieron a uretroplastia primaria. La uretroplastia primaria para algunos autores ofrece un panorama desalentador Koraitim y col. en 2.419 casos reportaron una alta incidencia de secuelas posquirúrgicas incontinencia en un $21 \%$ de los pacientes, impotencia en el 56\% y formación de estenosis hasta en el $49 \%$ de los pacientes ${ }^{8}$, sin embargo otros autores reportan tasas mas exitosas con tasas de incontinencia cercanas al $17,7 \%$, impotencia cercana al 33,3\% y formación de estenosis del 22,4\%2,3; Avanoglu y Balkan compararon los resultados de la uretroplastia primaria con el reparo tardío en niños encontrando que la uretroplastia primaria se asocia a menos estenosis en forma significativa ${ }^{9.10}$; la presencia de estenosis para los pacientes sometidos a uretroplastia primaria en nuestro estudio fue del $32 \%$.

En nuestros pacientes sometidos a uretroplastia primaria se encontró un menor riesgo de desarrollar infección, obstrucción y estenosis en comparación con aquellos pacientes que se les realizo reparo tardío.

La impotencia se reporta en un 33\% de los pacientes con trauma de uretra y la incontinencia en un $17 \%{ }^{7}$, en nuestro grupo el $10 \%$ de los pacientes presento impotencia y el 14\%, incontinencia, sin embargo, no logramos encontrar una diferencia significativa entre abordajes quirúrgicos para estas dos complicaciones. con un OR no significativo de un rango amplio que no nos permite inclinarnos hacia una de las 2 técnicas.
La lesión del cuello vesical ha sido reportada por Podesta como uno de los principales factores asociados a la incontinencia mas que la técnica quirúrgica como tal , en nuestro grupo de pacientes el 1,6\% (5 pacientes) presentaron lesión del cuello vesical, en ninguno de ellos se reporto incontinencia y solo 1 de ellos $(2,3 \%)$ presento impotencia. La presencia de disfunción eréctil probablemente sea mayor de lo que se reporta en la literatura dada la variabilidad de criterios con la que se define. En nuestros pacientes el porcentaje de presentación concuerda con lo reportado en la literatura ${ }^{3,7,8} \mathrm{sin}$ embargo, la corta edad de los pacientes y el corto periodo de seguimiento no permiten evaluar dicha variable en forma fidedigna.

En nuestra serie, el reparo tardío se realizo en el $30,2 \%$ de los pacientes, en series mas grandes de pacientes, como la reportada por Koraitim con 508 pacientes encontraron hasta un 97\% de estenosis, un $4 \%$ de incontinencia y hasta un 19\% de impotencia. En nuestros pacientes estas se presentaron en $20,9 \%$ ( 9 pacientes), $7 \%$ (3 pacientes) y $2,3 \%$ ( 1 paciente) respectivamente lo cual es concordante con la literatura ${ }^{11,12}$.

El manejo con dilataciones seriadas y progresivas en nuestros pacientes con estenosis uretral fue exitoso y en promedio se realizaron 4.5 dilataciones por paciente por paciente, solo 2 pacientes requirieron reintervenciones para la corrección de la estenosis, uno de ellos fue el único paciente a quien en este estudio se le realizo técnica de cateterización retrograda, este paciente requirió 2 reintervenciones y presentó complicaciones renales, asociadas hidronefrosis, hipertensión arterial y litiasis vesical, adicionalmente, la literatura reporta que la cateterización retrograda se asocia con un porcentaje de estenosis cercano al 50\%, impotencia e incontinencia y actualmente se ha remplazado por la colocación de catéteres vía endoscopia ${ }^{7,12}$.

Nuestros pacientes tuvieron estancias hospitalarias prolongadas con una media de 35,9 días lo que se relaciona principalmente con las lesiones asociadas un $76.7 \%$ presento fractura de fractura de pelvis y un $7 \%$ fracturas complejas de las extremidades que requirieron múltiples procedimientos quirúrgicos ortopédicos aumentando la estancia hospitalaria. El promedio de estancia hospitalaria en los pacientes con uretroplastia primaria fue de 32 días y para el grupo de cistotomía y reparo tardío de 34 días. 
El tiempo medio de seguimiento fue de 30,6 meses similar a lo encontrado en los diferentes estudios. Seguimientos a largo plazo serian ideales y necesarios. Para ver la respuesta de estos pacientes en la vida adulta; de igual forma estudios prospectivos que permitan estudios analíticos.

\section{CONCLUSIONES}

El manejo quirúrgico de la población pediátrica con trauma uretral continúa siendo controvertido, se requiere un ensayo clínico con seguimiento y resultados a largo plazo.

El mecanismo traumático y en especial el trauma cerrado con fractura de pelvis asociada son la presentación más frecuente.

La estenosis de uretra es la complicación que se presenta más frecuentemente.

Los diferentes abordajes quirúrgicos utilizados presentan tasas de complicación y de morbilidad a mediano plazo similares. El procedimiento de elección debe ser individualizado de acuerdo a las características del paciente, la extensión del daño uretral, las lesiones asociadas y la experiencia del cirujano en el manejo de dicha patología. Sin embargo en nuestro estudio encontramos que los pacientes en los cuales se realizo uretroplastia primaria presentaron un menor riesgo de infección, estenosis y obstrucción.

\section{Agradecimientos}

Sección de cirugía y urología pediátrica de la Universidad de Antioquia y Hospital Universitario San Vicente de Paúl.

\section{REFERENCIAS}

1. Holland AJ, Cohen RC, McKertich KM, Cass DT. Cass. Urethral trauma in children. Pediatric Surgery International. 2001;17(1):58-61.

2. Podestá ML, Medel R, Castera R, Ruarte A. Immediate management of posterior urethral disruptions due to pelvic fracture: Therapeutic alternatives. The Journal of Urology. 1997 Apr; 157 (4):1444-1448.

3. Onen A, Oztürk H, Kaya M, Otçu S. Long term outcome of posterior urethral rupture in boys: a comparison of different surgical modalities. Urology. 2005 Jun;65(6):1202-1207.

4. Upadhyaya M, Freeman NV. Manegement of traumatic urethral disruption in children: Oman experience, 1988-2000. Journal of Pediatric Surgery. 2002 Oct;37(10):1451-1455.

5. Mark SD, Keane TE, Vandemark RM, Webster GD. Impotence following pelvic fracture urethral injury: incidence, aetiology and management. British Journal Urology, 1995 Jan;75(1):62-64.

6. Chapple C, Barbagli G, Jordan G, Mundy AR, Rodrigues-Netto N, Pansadoro V, McAninch JW. Consensus statement on urethral trauma. British Journal Urology International, 2004 Jun; 93(9):11951202.

7. Nerli RB, Koura AC, Ravish IR, Amarkhed SS, Prabha V, Alur SB. posterior urethral injury in male children: long-term follow up. Journal of Pediatric Urology, 2008 Apr;4(2): 154-159.

8. Koraitim MM. Pelvic Fracture urethral injuries: the unresolved controversy. Journal of Urology, 1999 May;161(5):1433-1441.

9. Avano囚lu A, Ulman I, Herek O, Ozok G, Gökdemir A. Posterior urethral injuries in children. British Journal Urology, 1996 Apr;77(4): 597-600.

10. Balkan E, Kilic N, Dogruyol H. The effectivennesof early primary realignament in children with posterior urethral injury. International Journal of Urology. 2005 Jan;12(1):62-66.

11. Koraitim MM. Post-traumatic posterior urethral strictures in children a 20 yr experience. Journal of urology. 1997 Feb;157(2):641-645.

12. Orabi S, Badawy H, Saad A, Youssef M, Hanno A. Post-traumatic posterior urethral stricture in children: How to achive a successful repair. Journal of Pediatric Urology. Orabi S, Badawy H, Saad A, Youssef M, Hanno A.

Correspondencia autor: Dr. Jorge A. Martínez Montoya Sección de Cirugía y Urología Pediátrica. Facultad de Medicina. Universidad de Antioquia. Medellín. Colombia E-mail autor: cxpediatrica@medicina.udea.edu.co. Información artículo: Original - Pediatría Trabajo recibido: julio 2008

Trabajo aceptado: agosto 2008 